IOS Press

\title{
Thesis
}

\section{Interacting with smart environments: Users, interfaces, and devices}

\author{
Luigi De Russis \\ Department of Control and Computer Engineering (DAUIN), Politecnico di Torino, Italy \\ E-mail: luigi.derussis@polito.it
}

Keywords: Human-computer interaction, eye tracking, smart environments, wearable computing, green attitude, end-user development

On March, 20th, 2014, Luigi De Russis defended his Ph.D. dissertation entitled "Interacting with Smart Environments: Users, Interfaces, and Devices” at Politecnico di Torino, Italy. The research was supported by Fondazione CRT under the Lagrange project. ${ }^{1}$

Luigi De Russis defense took place in a publicly open presentation at the Department of Control and Computer Engineering (DAUIN) of Politecnico di Torino, and he was able to comment on every question raised by the assessing committee. The thesis was supervised by Prof. Fulvio Corno, and the dissertation committee was composed by Prof. Paolo Montuschi (Politecnico di Torino, Italy), Prof. Filippo Lanubile (Università degli Studi di Bari, Italy), and Prof. Alberto Bosio (LIRMM Université de Montpellier, France).

The full text of the thesis, licensed under the Creative Commons Attribution-NonCommercial 4.0 International License, is available on the Publication Open Repository of the Politecnico di Torino at http://porto.polito.it/2536887/.

\section{Thesis summary}

A Smart Environment is an environment enriched with disappearing devices, acting together to form an "intelligent entity". In such environments, the com-

\footnotetext{
${ }^{1}$ http://www.progettolagrange.it/en/, last visited on December 3, 2014
}

puting power pervades the space where the user lives, so it becomes particularly important to investigate the user's perspective in interacting with her surrounding. Interaction, in fact, occurs when a human performs some kind of activity using any computing technology: in this case, the computing technology has an intelligence of its own and can potentially be everywhere. There is no well-defined interaction situation or context, and interaction can happen casually or accidentally. All interactions, thus, should be as unobtrusive and "natural" as possible: users have to interact with the smart environment in different, explicit and implicit, alternative or combined ways, to ensure a robust and natural interaction. Moreover, users should not perceive the system as an opponent, an "entity" whose goal is to reduce their autonomy and control over the environment.

Up to now, the main focus of the smart environments research area has been technology-driven. Userrelated research in this field typically focuses on activity recognition, considering users localization and movements inside the environment and taking into account their privacy and security. Emerging trends encompass distributed user interfaces, systems to promote users' behavior changes, natural interaction, and applications for keeping users as autonomous as they desired in controlling their smart environment.

This thesis explores some of these trends, with the main and final goal to improve the interaction between users and smart environments (see Fig. 1 for an 


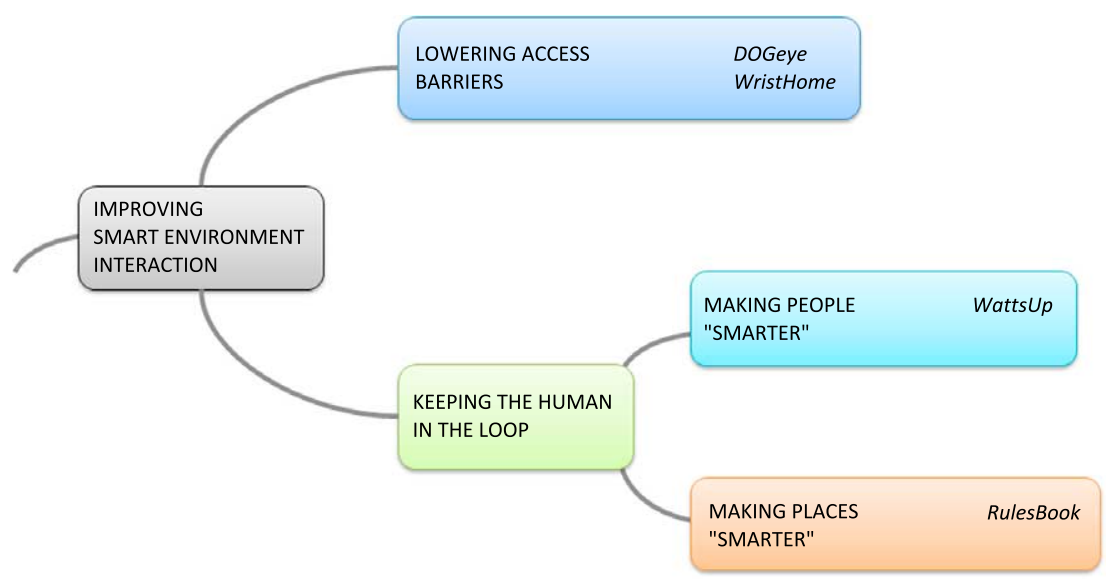

Fig. 1. Contributions of the thesis.

overview). To reach such a goal, four approaches are being tackled, in different domain areas, targeting the home as the envisioned environment. They follow two different but parallel paths: improving smart environment interaction by lowering access barriers and by keeping the human in the loop.

Each approach is associated to a specific software application that exemplify and realize it. The applications explore four different paradigms in their paths.

Universal Access for lowering access barriers is introduced by DOGeye [1], a multimodal eye-based application for home management and control, based on state-of-the-art technologies in both eye tracking and home control, and aimed at people with severe and evolving impairments, such as ALS (Amyothrophic Lateral Sclerosis).

Wearable Computing for lowering access barriers is presented by WristHome [2,3], a system for turning existing wrist-worn devices into flexible home access points by exploiting a modular architecture independent from the specific watch device and from the underlying home automation system.

Activity Delegation for making places "smarter" and keeping the user in control is presented by RulesBook, a mobile application for the creation of rules [4] and context-aware applications by end users. The objective here is to let the user maintain the desired autonomy in her home; a common way to realize this goal is by explicitly delegating some tasks to the smart environment, thus perceiving it as useful and cooperative.

Behavior Change for Energy Saving for making people more "aware" is exhibited by WattsUp, a system for informing users about their energy consumption patterns, and by suggesting more efficient and "green" behaviors. Such a system is supported by a user survey [5], distributed online between September 2010 and January 2011, and completed by 992 people.

This dissertation is mainly oriented toward users and their interactions with the overall system, and not toward the intelligent part. This lets the applications be general enough to be easily replicated with various intelligent systems, without invalidating the obtained results.

\section{References}

[1] D. Bonino, E. Castellina, F. Corno and L. De Russis, DOGeye: controlling your home with eye interaction, Interacting with Computers 23(5) (2011), 484-498.

[2] L. De Russis, D. Bonino and F. Corno, The smart home controller on your wrist, in: Proc. of the 2013 ACM Conference on Pervasive and Ubiquitous Computing Adjunct Publication, UbiComp '13 Adjunct, ACM, New York, NY, USA, 2013, pp. 785792.

[3] D. Bonino, F. Corno and L. De Russis, dWatch: a personal wrist watch for smart environments, Procedia Computer Science 10 (2012), 300-307, ANT 2012 and MobiWIS 2012.

[4] D. Bonino, F. Corno and L. De Russis, A user-friendly interface for rules composition in intelligent environments, in: $\mathrm{Ad}$ vances in Intelligent and Soft Computing, P. Novais, D. Preuveneers and J.M. Corchado, eds, Ambient Intelligence - Software and Applications, Vol. 92, Springer, Berlin, Heidelberg, 2011, pp. 213-217.

[5] D. Bonino, F. Corno and L. De Russis, Home energy consumption feedback: a user survey, Energy and Buildings 47 (2012), 383-393. 